COMMENTAIRE

\section{Agir contre les inégalités sociales de santé}

\author{
Tentative d'explications de l'immobilisme \\ des autorités de la santé publique québécoise
}

Valéry Ridde, PhD (cand), Msc, DESS

L e Québec est une des provinces canadiennes reconnues pour leurs politiques sociales progressistes. ${ }^{1}$ La santé publique québécoise a été précurseur d'une prise en compte des déterminants sociaux de la santé et intervient dans la lutte contre la pauvreté. ${ }^{2}$ Compte tenu de l'ampleur des inégalités sociales de santé au Québec, on s'attendrait à ce que leur réduction, à l'instar de quelques pays européens, devienne un nouveau cheval de bataille des acteurs de la santé publique. Or, l'étude du nouveau programme de santé publique 2003-2012, pris comme exemple "paradigmatique ", montre qu'il n'en est rien. Nous proposons trois hypothèses pour expliquer cet immobilisme.

Constater des inégalités de santé entre des individus ou des groupes de personnes dans une société fait directement référence à l'absence de justice sociale. La mort prématurée de certains et la vie prolongée d'autres est une constatation irréfutable des inégalités, dont la définition que nous retenons est : "il y a inégalité à partir du moment où une différence, une disparité se manifeste à propos d'un objet dont la possession est recherchée, d'un objet socialement valorisé. ${ }^{3}$ Rawls $^{4}$ disait que la justice est la première vertu et Descartes affirmait que "la conservation de la santé, [...] est sans doute le premier bien et le fondement de tous les autres biens de cette vie. " La définition de la santé retenue est celle promue par l'OMS, soit une santé globale; 6 cependant, pour illustrer l'existence (et la persistance) des inégalités de santé, nous sommes contraint - compte tenu de la disponibilité des travaux et faute de place - de nous limiter aux données de mortalité puisque "la différence dans la durée de vie est l'expression synthétique de l'ensemble des inégalités sociales. ${ }^{7}$

\section{Les inégalités de santé : constats et origines}

Au Québec, l'utilisation d'un indice de "défavorisation " montre que l'espérance de vie totale à la naissance est supérieure de 5,2 ans pour les personnes appartenant au quintile le plus favorisé (80,9 ans) par rapport au plus défavorisé (75,7 ans). ${ }^{8}$ L'écart est plus grand encore (14 ans) lorsque l'on compare l'espérance de vie sans incapacité entre ces mêmes quintiles. Le taux de mortalité foeto-infantile est de 11,1 pour 1000 lorsque les mères disposent d'une scolarité inférieure à 10 années alors qu'il est de 6,4 pour 1000 pour celles ayant étudié 14 années et plus. ${ }^{9}$ À Montréal, malgré l'amélioration globale de la santé des habitants, l'écart d'espérance de vie entre quartier atteint 13,5 ans chez les hommes et 8 ans chez les femmes. ${ }^{10}$ Dans la région urbaine de Montréal, le taux de mortalité dû à des maladies cardiaques est de 317 pour 100000 dans un quartier pauvre comparativement à 126 dans un quartier riche voisin. ${ }^{11}$

Contrairement à Rousseau, il est temps de comprendre que les inégalités de santé ne sont pas naturelles, ${ }^{12}$ les déficiences de naissance sont celles offrant le moins de variation entre

Département de médecine sociale et préventive, Faculté de médecine, Université Laval

Correspondance et demandes de réimpression : Valéry Ridde, Département de médecine sociale et préventive, Faculté de médecine, Université Laval, Pavillon de I’Est, Québec (Québec) G1K 7P4, Tél : 418-656-2131, poste 12658, Téléc. : 418-656-7759, Courriel : valery.ridde.1@ulaval.ca.

Remerciements : L'auteur tient à remercier Michel Vézina et Michel O’Neill pour leurs précieuses critiques d'une version antérieure de cet article, ainsi que les deux évaluateurs de la Revue. Une partie de cette réflexion a été effectuée grâce à I'implication de l'auteur au sein de l'équipe de recherche pluridisciplinaire sur les inégalités sociales de santé dirigée par Maria De Koninck. les catégories sociales. ${ }^{13}$ Les inégalités de santé sont la résultante d'un processus subtile et complexe d'inégalités sociales, d'incorporation des inégalités dans les corps, dit Fassin. ${ }^{12}$ Pour le dire autrement, les disparités devant la mort et la maladie sont l'aboutissement d'un mécanisme

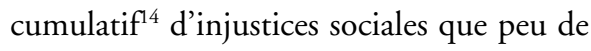
personnes ne paraissent vouloir remettre en cause.

Des actions contre la pauvreté, des réflexions et des lois sur les inégalités, mais rien de concret

Le Québec et ses acteurs de la santé publique sont préoccupés par la lutte contre la pauvreté. Nous avons dit que cette province est reconnue pour ses politiques sociales efficaces. ${ }^{1}$ La Politique de la santé et du bien-être de 1992 a été appréciée mondialement pour avoir mis l'accent sur les déterminants sociaux de la santé. ${ }^{2}$ À la suite de quoi, de nombreuses interventions ont été implantées pour lutter contre la pauvreté, plus tard notamment à travers les Priorités nationales de santé publique 1997-2000. Mais agir contre la pauvreté et ses effets ne règle pas le problème des inégalités de santé. Cette focalisation sur la pauvreté au détriment des inégalités de santé n'est pas nouvelle, tel qu'en témoigne le cas français. ${ }^{15,16}$ On agit sur l'absolu au détriment du relatif. Ainsi, contrairement à certains pays européens, aucune politique publique spécifiquement consacrée à la lutte contre les inégalités sociales de santé n'a émergé au Québec. Les décideurs n'ont pas encore véritablement compris l'ampleur du problème car «la permanence de son invisibilité dans l'espace politique en constitue un trait essentiel. ${ }^{17}$ Certes, il y a eu quelques avancées.

Quelque temps après le rapport concernant les inégalités de santé à Montréal en $1998,{ }^{18}$ un comité ministériel sur la réduction des inégalités de santé liées à la pauvreté a été mis en place. De septembre 1999 à mai 2000 une consultation provinciale a été organisée. Des initiatives de recherche sur les inégalités de santé ont cours à Montréal, ${ }^{19}$ Québec $^{20}$ ou en Mauricie. ${ }^{21}$ De surcroît, le législateur québécois a récemment entériné la nécessité d'agir. La loi 36 de santé publique impose au ministère de la santé d'organiser : "les actions [...] qui peuvent influencer les inégalités de santé. " ${ }^{22}$ 
La loi 112 a été votée pour lutter contre la pauvreté et l'exclusion sociale; elle fait référence à la réduction des inégalités sociales. ${ }^{23}$

Mais concernant les inégalités de santé, depuis lors, aucune stratégie ministérielle d'actions concrètes n'a été rendue publique.* Reprenant les étapes de Whitehead ${ }^{24}$ concernant le continuum d'actions liées à la réduction des inégalités sociales de santé, nous pourrions avancer que le Québec n'en est qu'aux premières étapes. Le problème est mesuré, plus ou moins reconnu et certains ont attiré l'attention de la population sur cette question, mais rien n'est réellement fait. Prenons un exemple pour expliciter cette affirmation.

\section{Le nouveau programme national de santé publique du Québec}

Le programme national de santé publique (PNSP), en tant que dernière politique publique $^{25}$ de santé, est un exemple "paradigmatique " de cet état d'esprit. Ce programme reconnaît qu'il existe des inégalités de santé et que certains sousgroupes de la population sont moins nantis que d'autres. Dans la discussion des principaux enjeux contemporains de santé publique, les auteurs notent que "le quatrième et dernier défi réside dans la réduction des inégalités en ce qui concerne la santé. " ${ }^{26} \mathrm{Il}$ se conforment ainsi aux exigences prônées par la loi.

Permettons-nous d'abord une remarque sémantique et épistémologique. Les experts ont repéré des sous-groupes de la population moins nantis que d'autres. Ces groupes sont qualifiés de "vulnérables.$^{26}$ Le terme employé, " groupes vulnérables ", est on ne peut plus stigmatisant tant le mot vulnérable dispose d'une connotation que Lupton ${ }^{27}$ nommerait de "victim blaming ". L'emploi de ce vocable renvoie à des déterminants individuels des inégalités de santé et non pas à des déterminants sociaux et politiques. Le processus de production de cette vulnérabilité, comme il semble que cela soit souvent le cas, ${ }^{28}$ est éludé; ce qui n'est pas sans nous rappeler les problèmes épistémologiques liés à l'usage du terme précarité. ${ }^{29}$ Des programmes alimentaires à

Seul le rapport du comité ministériel sur la réduction des inégalités de santé et de bien-être, datant de décembre 2002, a été publié le 26 septembre 2003, mais uniquement en version électronique. destination des enfants pauvres des provinces atlantiques du Canada, plein de bonnes volontés mais usant de ce type d'approche, ont eu pour conséquences de stigmatiser les bénéficiaires et de reproduire, plutôt que réduire, les inégalités. ${ }^{30}$

Les responsables du PNSP affirment que la réduction des inégalités de santé est un des quatre enjeux majeurs de la santé publique québécoise. Cependant, lorsque l'on regarde en détail les 87 objectifs du programme, aucun (0) n'est exprimé en fonction d'écarts à réduire entre des sousgroupes de la population. L'unique domaine (le développement des communautés) évoquant clairement " des activités qui réduisent les inégalités de santé, ${ }^{26}$ est le seul dont aucun objectif n'a été préalablement déterminé.

\section{Trois hypothèses pour expliquer l'immobilisme de la santé publique}

Ainsi, l'obligation légale de réduire les inégalités de santé et la précision que cela constitue une priorité de santé publique au Québec, paraissent, au regard du contenu du PNSP, encore cantonnées au plan discursif. Les inégalités de santé sont présentes mais rien n'est proposé pour les résorber, à tout le moins tendre vers la réduction des écarts. Pour rendre intelligible cette situation, nous oserions avancer une triple hypothèse.

La première hypothèse concerne la tendance mondiale de vouloir absolument planifier les activités et formuler les politiques publiques sur des données probantes. En agissant ainsi, les responsables québécois se conforment aux exigences de la loi de santé publique puisque seules les actions réputées efficaces ont été retenues. Toutefois, vouloir implanter des activités visant la réduction des inégalités uniquement à l'aide de données probantes est difficile et certainement pas complètement pertinent. Difficile, car l'état des connaissances actuelles à ce propos est infime. ${ }^{31,32}$ Même en Angleterre, un des pays phares pour ce type d'actions publiques, le groupe d'évaluation des propositions de projets à la suite de la commission Acheson ${ }^{33}$ a été très surpris de la quasi-absence de fondements empiriques dans les actions proposées. ${ }^{34}$ Macintyre ${ }^{35}$ avance quatre raisons pour expliquer l'absence de données probantes : les données ne sont pas différenciées par catégorie, les ressources se focalisent plus sur la recherche et la définition des problèmes que sur la résorption de ces derniers, ${ }^{*}$ le degré d'attente (" evidence-based ", " essais randomisés ") des résultats d'évaluation des interventions favorise le statut quo, le suivi n'est pas organisé. Mackenbach ${ }^{36}$ ajoute une cinquième raison : l'absence de rapport d'évaluation d'interventions efficaces dans les bases de données classiques. Les chercheurs sont en partie responsables de cette inertie car dans un article de 1997, le seul à notre connaissance traitant de la méthodologie pour évaluer des interventions visant la réduction des inégalités de santé, le paradigme positiviste est largement explicite et les devis expérimentaux ont une grande place, sinon la seule. ${ }^{37}$

Notre deuxième hypothèse se réfere aux acteurs de la santé publique québécoise. La seule fois où la réduction des inégalités est explicitement évoquée, c'est dans le domaine des activités de développement des communautés. Ce domaine d'intervention émerge telle une incongruité pour la santé publique. Lorsque l'on évoque la structure du programme dans la première section du document, six domaines d'activités sont présentés, mais pas celui du développement des communautés. Il est conçu simplement comme une stratégie d'intervention et non comme un domaine d'activités. Ainsi, le programme se conforme aux domaines dévolus à la santé publique traditionnelle et dénote le malaise des acteurs à vouloir intégrer l'approche communautaire comme un domaine d'activité. Les préoccupations des experts sont dirigées vers d'autres sphères. Il suffit pour cela de faire l'exercice de relier le nombre d'objectifs par domaine du PNSP avec le nombre de spécialistes ayant participé à la rédaction du document. Le développement des communautés est placé comme un domaine car, s'il est présenté comme une stratégie au début du document, par la suite, il est proposé dans le corps du texte tel un domaine d'intervention (p.70). Il serait intéressant (mais impossible ?) d'ajouter au tableau I une ligne

Bien que dans un article de 1997, Mackenbach et al. perçoivent un "shift towards a more action oriented climate» (réf. 37, p. 359) 
TABLEAU I

Objectifs et experts en fonction des domaines d'intervention du programme de santé publique, adapté de réf. 26

Nombre d'objectifs Nombre d'experts

Nombre d'objectifs Nombre d'experts

correspondant au fardeau de mortalité lié à ces sept domaines différents.*

Enfin, notre dernière hypothèse concerne l'absence d'objectifs dans le seul domaine où la réduction des inégalités est explicitement nommée. Il est possible de croire que l'idée première est de faire en sorte que les objectifs soient déterminés par les communautés elles-mêmes. Ce qui nous paraît complètement louable et doit, croyons-nous, constituer la démarche d'avenir pour que la réduction des inégalités soit envisageable. ${ }^{38}$ En attendant que les équipes de recherche produisent des données probantes quant aux interventions efficaces et qu'une prise de conscience nationale émerge, des interventions et des recherches-actions concertées avec les communautés locales doivent être mises en œuvre, comme cela fut le cas par exemple à Detroit. $^{39}$

\section{Conclusion}

Si la lutte contre les inégalités sociales de santé ne relève pas uniquement du champ de la santé publique, elle devrait au moins devenir une priorité des politiques publiques de santé. Il faut que cela se traduise, au minimum par une description de la situation sanitaire sensible aux sousgroupes de la population, mais surtout par la formulation des activités à mettre en œuvre. Pour cela, il faudrait que les interventions de santé publique soient moins traditionnelles (technocratique) et plus avant-gardistes (communautaire). ${ }^{40}$ Les objectifs à atteindre doivent être formulés en conséquence et les indicateurs de performance doivent être sensibles aux écarts entre les sous-groupes. En 1998, l'Europe s'est fixée l'objectif de réduire d'au moins un quart, d'ici 2020, l'écart de santé entre les groupes socioéconomiques. ${ }^{41}$ L'Angleterre vient de

\footnotetext{
* En outre, ce tableau permet de mettre en exergue le caractère fragmentaire du champ de la santé publique malgré les discours de l'interdisciplinarité.
}

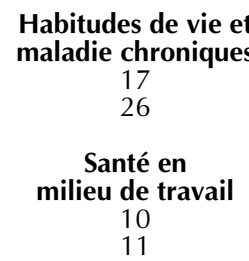

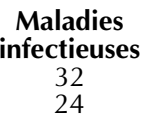

Développement

des communautés

0 réaliser une revue interministérielle des actions efficaces. ${ }^{42}$ Ses experts ne planifient pas uniquement à l'aide de données probantes mais également au regard d'une "governmental vision ". ${ }^{43}$ Les acteurs de la santé publique québécoise ne pourraient-ils pas en faire autant?

\section{REFÉRENCES}

1. Développement des ressources humaines Canada. Les statistiques de 2000 sur le faible revenu selon la mesure du panier de consommation. Ottawa : Direction générale de la recherche appliquée, Politique stratégique, Développement des ressources humaines Canada; Mai 2003, SP-569. 03-03F 2003.

2. Gouvernement du Québec. La politique de la santé et du bien-être. Québec : Ministère de la santé et des services sociaux, 1992.

3. Aïach P, Carr-Hill R, Curtis S, Illsley R. Les inégalités sociales de santé en France et en GrandeBretagne. Paris : INSERM. La documentation française, 1987.

4. Rawls J. Théorie de la justice. Paris : du Seuil, 1993.

5. Descartes R. Discours de la méthode, avec une notice biographique. Paris : Larousse.

6. Rootman I, Raeburn J. The concept of health. In: Rootman I (Ed.), Health Promotion in Canada. Toronto, ON: W.B. Saunders, 1994;5671.

7. Aïach P. De la mesure des inégalités : enjeux sociopolitiques et théoriques. Dans : Leclerc A, Fassin D, Grandjean H, Kaminski M, Lang T (Eds.), Les inégalités sociales de santé. Paris : Inserm - La découverte, 2000;83-92.

8. Pampalon R, Raymond G. Un indice de défavorisation pour la planification de la santé et du bien-être au Québec. Maladies chroniques au Canada 2000;21(3):113-22.

9. Chen J, Fair M, Wilkins R, Cyr M. Materna education and fetal and infant mortality in Quebec. Fetal and Infant Mortality Study Group of the Canadian Perinatal Surveillance System. Health Rep 1998;10(2):53-64 (Eng); 57-70 (Fre).

10. Lessard R. Nouvelles perspectives sur la santé des Montréalais : une action commune pour réduire les inégalités. Policy Options 2003;March:41-45.

11. O'Loughlin JL, Paradis G, Gray-Donald K, Renaud L. The impact of a community-based heart disease prevention program in a lowincome, inner-city neighborhood. Am J Public Health 1999;89(12):1819-26.

12. Fassin D. L'espace politique de la santé, essai de généalogie. Paris: Presses Universitaires de France, 1996.

13. Ravaud J-F, Mormiche P. Handicaps et incapacités. Dans : Lang T (Ed.), Les inégalités sociales de santé. Paris : INSERM-La découverte, 2000;295-314

14. Bihr A, Pfefferkorn R. Déchiffrer les inégalités, 2 éd. Paris : Syros, 1999.
15. Joubert M, Chauvin P, Facy F, Ringa V (Eds.), Précarisation, risque et santé. Paris : INSERM Questions en santé publique, 2001.

16. Leclerc A, Fassin D, Grandjean H, Kaminski M, Lang $\mathrm{T}$ (Eds.), Les inégalités sociales de santé. INSERM - La découverte, 2000.

17. Drulhe M. Fin de siècle et inégalités sociales de santé en France : l'amorce d'un débat ? Dans : Leclerc A, Fassin D, Grandjean H, Kaminski M, Lang T (Eds.), Les inégalités sociales de santé. Paris : INSERM - La découverte, 2000;41-51.

18. Direction de la santé publique. Rapport annuel 1998. Réduire les inégalités sociales de la santé. Montréal : Régie régionale de la santé et des services sociaux de Montréal-Centre, 1998.

19. Potvin L, Lessard R, Fournier P. Inégalités sociales de santé : un partenariat de recherche et de formation. Can I Public Health 2002;93(2):134-37.

20. De Koninck M, Clément M, Dubois L, LaRue A, Malenfant R, Lévesque $\mathrm{M}$, et al. Inégalités de santé et milieux de vie : déterminants sociaux en cause et leurs interactions. 2002.

21. Boisvert R, Pepin Y, Rocheleau L, Crosato S. Les inégalités de santé et de bien-être en Mauricie et au Centre-du-Québec : une analyse écologique. Régie régionale de la santé et des services sociaux de la Mauricie et du Centre-du-Québec. Groupe Vigie et qualité des services. Direction de la santé publique, 2000

22. Gouvernement du Québec. Projet de loi $n$ 36. Loi sur la santé publique. Québec, 2002.

23. Gouvernement du Québec. Loi visant à lutter contre la pauvreté et l'exclusion sociale. Québec : Assemblée Nationale, 18 décembre 2002.

24. Whitehead M. Diffusion of ideas on social inequalities in health: A European perspective. Milbank Q 1998;76(3):469-92, 306.

25. Lemieux V. L'étude des politiques publiques, les acteurs et leur pouvoir, 2ème éd. Québec : Les Presses de l'Université Laval, 2002.

26. Ministère de la Santé et des Services sociaux. Programme national de santé publique 2003-2012. Québec : MSSS, 2003.

27. Lupton D. The Imperative of Health: Public Health and the Regulated Body. London; Thousand Oaks, CA: Sage Publications, 1995.

28. Gendron S. La pratique participative en santé publique : l'émergence d'un paradigme [PhD Santé publique option promotion de la santé]. Montréal : Département de médecine sociale et préventive, 2001.

29. Appay B. Précarité, précarisation : réflexions épistémologiques. Dans : Joubert $\mathrm{M}$, Chauvin $\mathrm{P}$ Facy F, Ringa V (Éds.), Précarisation, risque et santé. Paris : INSERM - Questions en santé publique, 2001;15-27.

30. McIntyre L, Travers K, Dayle JB. Children's feeding programs in Atlantic Canada: Reducing or reproducing inequities? Can J Public Health 1999;90(3):196-200.

31. Stronks K. Generating evidence on interventions to reduce inequalities in health: The Dutch case. Scand J Public Health 2002;Suppl 59:20-25.

32. Gepkens A, Gunning-Schepers LJ. Interventions to reduce socioeconomic health differences: A review of the international literature. European I Public Health 1996;6:218-26. 
33. Acheson D. Independent Inquiry into Inequalities in Health Report. London, 1998.

34. Macintyre S, Chalmers I, Horton R, Smith R. Using evidence to inform health policy: Case study. BMJ 2001;322(7280):222-25.

35. Macintyre S. Evidence based policy making. BMJ 2003;326(7379):5-6.

36. Mackenbach JP. Tackling inequalities in health: The need for building a systematic evidence base. I Epidemiol Community Health 2003;57(3):162.

37. Mackenbach JP, Gunning-Schepers LJ. How should interventions to reduce inequalities in health be evaluated? J Epidemiol Community Health 1997;51(4):359-64.

38. Ridde V. Quelles interventions pour lutter contre les inégalités sociales de santé ? Québec: Université Laval, Équipe de recherche "Inégalités de santé et milieux de vie"; Juin 2003

39. Schulz A, Israel B, Parker E, Lockett M, Hill Y, Wills R. The East Side Village Health Worker Partnership: Integrating research with action to reduce health disparities. Public Health Report 2001;116(6):548-57.

40. Conill EM, O’Neill M. La notion de santé communautaire : éléments de comparaison internationale. Can J Public Health 1984;75(3):166-75.

41. Zollner H. National policies for reducing social inequalities in health in Europe. Scand J Public Health 2002;Suppl 59:6-11.

42. Department of Health. Tackling Health Inequalities - Cross-Cutting Review. London: DOH, 2002.

43. Nutbeam D. Implementing policies across central government. Allocution lors du colloque "UK Health Equity Network, Health inequalities: Evidence, policy and implementation" 5 July, 2002, London.

Reçu : 7 mai 2003

Accepté : 24 novembre 2003
Science - Programmes - Collaboration

\section{$6^{\text {th }}$ Canadian Immunization Conference}

December 5-8, 2004,

Palais des congrès de Montréal; Montréal, Québec, Canada

Mcotretal is this yoar's site for Canada's most importain conference on immanization.

$$
\text { Picase join wes! }
$$

- Organiwed by

The Immunization and Respiratory Infections Division. Healch Canado, and the Canadian Pardiatrie Society

\section{- Conference Goals}

Frovkle delepules with cuning edge information on immunizs. bon sciesce, policy, programmes and practice, and a foram for

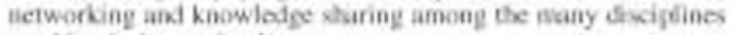
working in immanization

- Conference participants will share hooulcdge an

Nes vaccine bechnalogies

Vaccines and the challenge of emerging intectioms

Imrnunization for First Naxinns and other specifie popefation: Information techrology and immunization

Best prastices in imanumisation

influencing public policy and informed decisios-making

The National Immunization Strategy

Cantads's cole in stotal immunization

- Who should attend?

$$
\begin{aligned}
& \text { Public health physicians and munses } \\
& \text { Primary care physicians and nurses } \\
& \text { Speciahty perysicians and surse: } \\
& \text { Health professionsls in education } \\
& \text { Healsh pofessiceals in trab nimg } \\
& \text { Health promaters } \\
& \text { Researchers in vacesmology } \\
& \text { Phammacists } \\
& \text { Policy makess } \\
& \text { Studemts. } \\
& \text { Industry }
\end{aligned}
$$

This year, the scientific absiract compoeent of the Conference is teing enlarzod, with assustance from the Canadian Assnciation for Immunization Research and Evaluation (CAIRE).

Submissoms will he peer revieued and iceepeed absiracis mav be published. Visit the coeference Web site for more details.

This event is supported by the Canadian Pacdiatric Society as an Acendited Group Learning Activity (Section 1) as defined by the Maintenance of Cerrification Program of The Rogal College of Physicians and Surgeons of Canada.

An applicution for certification is pending with the Quetec Collewe of Family Physicians under the continuing medical educatios prognams.

Visit the Conference Web site for proecam updinos and sestritic alsomat information at:

hitp://wum.he-sc.pcea'pphb-daspapicnic-ecni

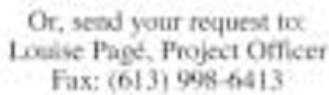

$$
\text { Fax: }(6) 3) 998-6413
$$

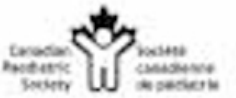

